\title{
Comparison of two full-field identification methods for the wedge splitting test on a refractory
}

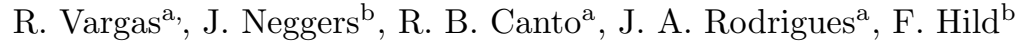 \\ ${ }^{a}$ DEMa - UFSCar, Rodovia Washington Luis, km 235, 13565-905 São Carlos-SP, Brazil \\ ${ }^{b}$ Laboratoire de Mécanique et Technologie (LMT) \\ ENS Paris-Saclay, CNRS, Université Paris-Saclay \\ 61 avenue du Président Wilson, 94235 Cachan, France
}

\begin{abstract}
Two full-field identification methods are applied to the Wedge Splitting Test (WST) to obtain crack tip positions, stress intensity factors (SIFs) and T-stress. The first method is based on Finite Element Model Updating (FEMU), and the second is integrated digital image correlation (IDIC). Both are applied to a simplified virtual experiment and then to a cyclic WST. The gray level residuals are used to assess which results are more trustworthy. Fracture energy analyses are performed to validate the estimated R-curves.

Keywords: Crack tip position, digital image correlation, finite element model updating, stress intensity factors, virtual test, crack propagation
\end{abstract}

\footnotetext{
${ }^{*}$ Corresponding author

Email address: hild@lmt.ens-cachan.fr (F. Hild)
}

Preprint submitted to Journal of European Ceramic Society

July 23, 2018 


\section{Introduction}

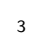

4

Refractory castables are ceramics that enable functional properties to be maintained in aggressive applications, generally at high temperatures [1. Not only the chemical composition but also raw material characteristics should be considered when designing new formulations since they affect the resulting phases, microstructures and thermomechanical properties [2]. Different compositions may be needed for the same application because of several thermomechanical loading steps during industrial processes [3, which spur innovations to improve performance such as increasing reactiveness during processing by using nanomaterials [2] or mimicking microstructures observed in nature [4]. In high risk applications, the material and mechanical properties should be well understood to better predict failures and thereby prevent accidents, while maximizing efficiency and lifetime [5].

The Wedge Splitting Test (WST) is a mechanical experiment allowing crack propagation to be assessed in (quasi)brittle materials [6, 7]. It leads to stable crack propagation by decreasing the elastic energy stored in the machine using a wedge and cylinders to apply the load [8]. A high fractured-area to volume ratio leads to achieving representative results for coarser microstructures at laboratory scales [7. The classical goal of WSTs is to obtain the fracture energy, by integrating the load vs. displacement curve and dividing by the projected fractured area [7]. Although important, the fracture energy itself is not the only information that can be extracted from WSTs. Recently, it is becoming common practice to seek more data from each of these tests to better understand the fracture process. Relationships between WST measurements with microstructures can be obtained 9], and various works have shown how different compositions affect crack propagation (with the WST) for magnesiaspinel [10, $\mathrm{Al}_{2} \mathrm{O}_{3}-\mathrm{C}$ [11], $\mathrm{Al}_{2} \mathrm{O}_{3}-\mathrm{MgO}-\mathrm{CaO}$ [12, and $\mathrm{MgO}-\mathrm{C}$ [13, 14] systems. It is also possible to identify fracture properties using measured load data and compare them with numerical simulations of the WST [15].

More experimental data can be acquired from WSTs via full-field measure- 
ments. Among them, Digital Image Correlation (DIC) enables displacement fields to be measured [16], and has been successfully used in crack propagation analyses [17, 18, 19, 20, 21, 22]. DIC was already applied to WSTs to analyze the strain fields for microcrack formation in magnesia refractories, when a spinel phase was added [23]. Similar ideas were used to quantify the fracture process zone of magnesia refractories, and highlight how microcracks tend to decrease the strength but increase the fracture energy [24, and to estimate the crack growth resistance [25]. Another interesting approach is to measure crack propagation while checking the discontinuities in the displacement field [26]. An integrated-DIC (IDIC) scheme to measure the R-curve behavior was also proposed [27] by considering closed-form solutions of one propagating crack in an elastic medium [28].

The aforementioned test [27] will be considered herein in order to compare two different approaches for estimating fracture mechanics parameters using full-field measurements. It is important to note that the hypothesis of one straight crack is reasonable in experiments with the presence of a groove on the propagating faces, as commonly used for the WST to ensure more straight crack propagation [29]. However, crack branches may occur in the WST [25] and it should be checked for each test. With both approaches investigated herein, this check is part of the methodology.

In this paper, a procedure based on the methodology used in Ref. [30] is applied to the WST. By using the outer measurements from DIC analyses as Boundary Conditions (BC) for a Finite Element (FE) analysis and using internal nodes for error estimators, it is possible to determine the crack tip position and calculate Stress Intensity Factors (SIFs), i.e., $K_{1}$ and $K_{2}$, and the T-stress. It will then be compared with IDIC. First, the experiment, the DIC principles and both methodologies that will be compared are introduced. It is followed by an analysis of a virtual experiment. Then an experimental study is performed to compare both methods. 


\section{Methods}

The two methods studied herein are summarized hereafter. Both of them were used independently to analyze various experiments with cracks 30, 31, 20, 27. However, they were never compared with the same data set, be they synthetic or from an actual experiment. The first method couples FE analyses and DIC measurements in order to determine the crack tip position, stress intensity factors and $T$-stresses [30]. It belongs to the class of finite element model updating techniques [32]. The second approach corresponds to integrated DIC [20, which is a standalone technique in comparison with the previous framework when applied to the analysis of cracked samples. Augmented Williams' series 28 are used, in particular, for the determination of the crack tip position [33, 34].

\subsection{Experiment}

The WST analyzed herein was performed on a class C, anti-erosive commercial refractory, with ultra low cement content, whose typical mineralogical composition consists of quartz, mullite, kyanite, $\beta$-cristoballite and alumina [27]. Water was added to the mixture up to $8.5 \mathrm{wt} \%$ of concrete. Drying was performed for $48 \mathrm{~h}$ in humid environment at room temperature followed by $24 \mathrm{~h}$ at $110^{\circ} \mathrm{C}$. A heat treatment was performed with a $1^{\circ} \mathrm{C} /$ min rate and kept at $500^{\circ} \mathrm{C}$ for $24 \mathrm{~h}$. The detailed chemical composition and the treatment of the material are reported in Ref. [27. Its processing and microstructure may lead to an increasing R-curve behavior, with weakly bonded grains and initiated microcracks due to anisotropic phases and differential thermal expansions.

The sample size is $100 \mathrm{~mm}$ in length, $100 \mathrm{~mm}$ in height and $72.5 \mathrm{~mm}$ in thickness. The geometry is shown in Figure 1 in which it is possible to see the contour of the sample and the loading devices (wedge, cylinders and blocks). Two grooves (i.e., lateral notches, see dashed line in Figure 11) are machined on two opposite faces of the sample to reduce the local thickness and guide the crack propagation vertically. The two zones where the splitting displacement is 
evaluated via DIC are also shown in Figure 1 as yellow boxes. Considering $\delta$ as the initial distance between both zones, the horizontal displacements measured on these regions are averaged and their difference accounts for the splitting displacement $\Delta \delta$, which will be reported in Section 4 .

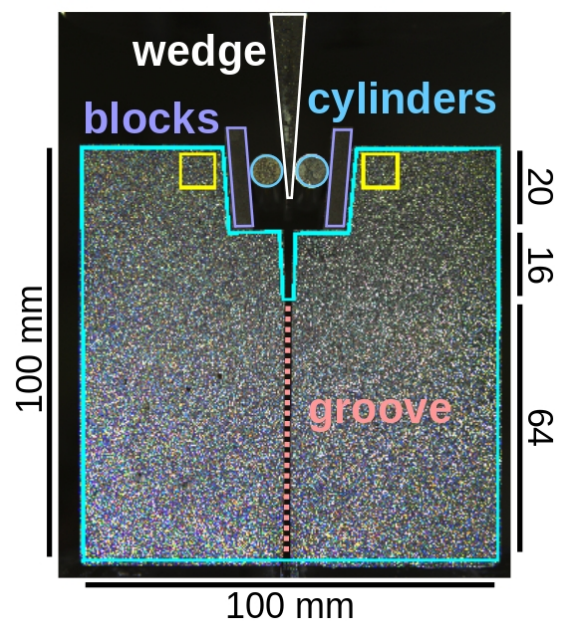

Figure 1: Detailed geometry of the wedge splitting test. The contour of the sample, including the pre-notch, is shown in cyan. The wedge, cylinders and blocks used to apply load are highlighted in white, blue and purple, respectively. The vertical groove in order to guide the crack vertically is shown with the dashed red line. The splitting displacement $\Delta \delta$ corresponds to the difference of the mean displacement of the two yellow boxes. The thickness of the specimen is $72.5 \mathrm{~mm}$. All dimensions are expressed in $\mathrm{mm}$.

The Young's modulus (E) and Poisson's ratio used for the investigated methods are equal to $17 \mathrm{GPa}$ (measured by the bar resonance method [35]) and 0.2, respectively. The test was driven by setting the velocity of the machine actuator to $1.3 \mu \mathrm{m} / \mathrm{s}$, and 313 pictures were taken for both faces of the specimen at a rate of one picture each $8 \mathrm{~s}$. The images were simultaneously acquired with two Canon T5 cameras with 28-135 mm lenses, with the illumination provided by LEDs. The 16-bit picture definitions are $2601 \times 1733$ pixels, with a dynamic range of approximately 60,000 gray levels. The imaged physical size of one pixel was $62 \mu \mathrm{m}$. A random speckle pattern was sprayed onto the specimen surfaces to increase the image contrast and improve the DIC resolutions. 
The 5-cycle loading curve of the experiment, which corresponds to the vertical force $F_{v}$ vs. vertical actuator displacement $\Delta h$, is shown in Figure 2, Further information on this test can be found in Ref. [27, and further characterization of the studied material, processing and microstructure in Refs. [36, 37.

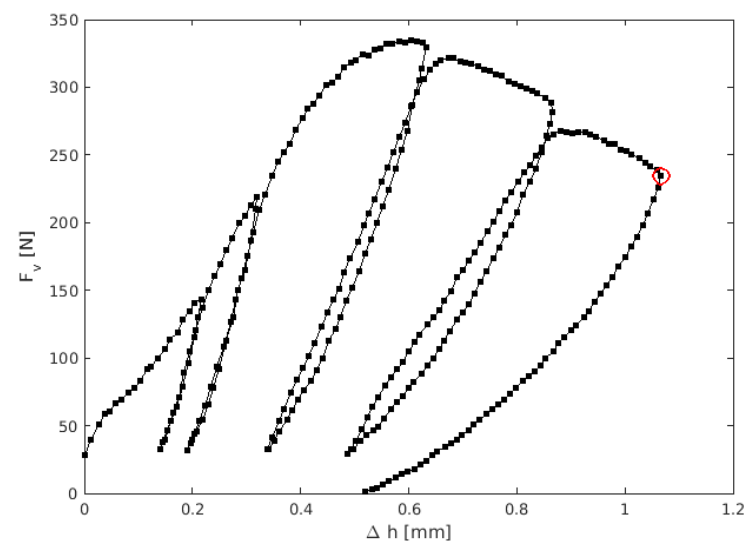

Figure 2: Loading history for the test analyzed in Section 4 Each point represents two image acquisitions (one per face) performed during the test for DIC analyses. The red circle depicts the picture acquisitions used to evaluate the strain fields shown in Figure 3

\subsection{Digital Image Correlation}

In global DIC, the displacement fields $\mathbf{u}(\mathbf{x})$ are measured by minimizing the L2-norm of the gray level residuals, $\phi^{2}$, between the image of the reference state $f$, and at the deformed state $g$

$$
\phi^{2}=\sum_{\mathrm{ROI}}[f(\mathbf{x})-g(\mathbf{x}+\mathbf{u}(\mathbf{x}))]^{2} .
$$

1 Searching for displacements at the pixel level is an ill-posed problem [38]. Parameterizations of the sought displacement $\mathbf{u}$ are used to regularize this problem and render the solution less affected by the acquisition noise

$$
\mathbf{u}(\mathbf{x})=\sum_{i=1}^{N} v_{i} \boldsymbol{\Psi}_{i}(\mathbf{x}),
$$

4 in which $v_{i}$ are the degrees of freedom, and $\boldsymbol{\Psi}$ shape functions (i.e., vector fields) that combine the displacement of a group of pixels in order to make the 
problem well-posed. The solution becomes

$$
\left\{\boldsymbol{v}_{\mathrm{DIC}}\right\}=\underset{\{\boldsymbol{v}\}}{\arg \min } \phi^{2}(\{\boldsymbol{v}\}),
$$

where $\left\{v_{\text {DIC }}\right\}$ is the column vector gathering all amplitudes $v_{i}$. If the actual kinematics of the problem is not well known a priori, $\boldsymbol{\Psi}_{i}$ can be chosen as finite element shape functions 39. In the present case, the DIC procedure is performed with 3-noded linear elements based on finite element discretization 40] and will be referred as T3DIC. In global approaches, the quality of the registration can be evaluated pixel-wise by computing the gray level residuals

$$
\rho_{\mathrm{T} 3}=f(\mathbf{x})-g\left(\mathbf{x}+\mathbf{\Psi}_{\mathrm{T} 3}\left(\mathbf{x},\left\{\boldsymbol{v}_{\mathrm{T} 3}\right\}\right)\right),
$$

where $\boldsymbol{\Psi}_{\mathrm{T} 3}$ is the vector containing the shape functions converting nodal to pixel displacements, which depends linearly on the amplitude $\left\{\boldsymbol{v}_{\mathrm{T} 3}\right\}$. In the present case, the T3DIC mesh is composed of 3-noded elements whose average edge length is equal to 58 pixels (or $3.6 \mathrm{~mm}$ ).

In the following, the global residual of DIC approaches will be compared. It is defined as the root-mean-square (RMS) gray level residual over the considered ROI

$$
\bar{\rho}_{\mathrm{T} 3}=\frac{R M S\left(\rho_{\mathrm{T} 3}\right)}{\Delta f},
$$

where $\Delta f$ is the dynamic range of the picture of the reference configuration

$$
\Delta f=\max _{\mathrm{ROI}} f-\min _{\mathrm{ROI}} f \approx 60,000 \text { gray levels. }
$$

The first step of any of the methods presented hereafter is to run T3DIC. It provides displacement fields that can be compared with FE analyses, but also allows the crack path to be chosen for integrated DIC [27. The maximum eigen strain field is selected in order to check the validity of the straight crack propagation assumption and the presence of a single macro-crack. The two faces of the sample are analyzed with a very fine mesh of average element length of 8.5 pixels (or $530 \mu \mathrm{m}$ ). Figure 3 shows the results for both faces for the last image before the final unloading (Figure 2). The standard uncertainty of the 


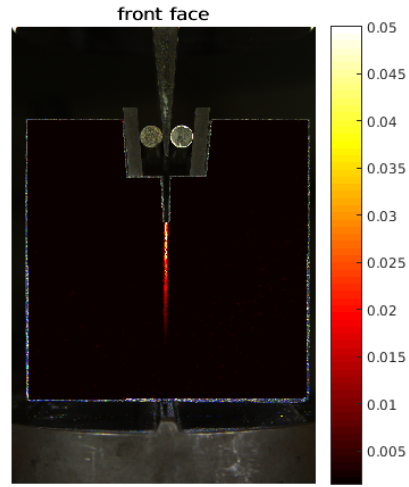

maximum eigen strain is of the order of $3 \times 10^{-4}$ and the minimum strain level in the color bars of Figure 3 is set to 3 times this value. It was determined by correlating the two pictures shot for the reference configuration on each face. Only one unique macro-crack is observed (guided by the groove).

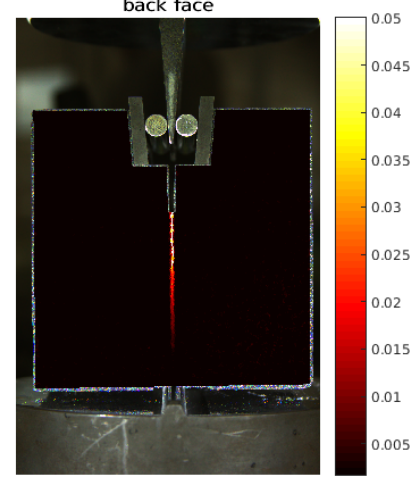

Figure 3: Maximum eigen strain fields for both analyzed faces for the image before the 5th unloading. No macro-crack branches are seen and consequently, the hypothesis of a straight crack propagation guided by the groove can be made.

\subsection{Method 1: Finite Element Model Updating}

The methodology described in this section is an adaptation of that proposed in Ref. [30] to the WST. DIC results are taken as Dirichlet boundary conditions and FE formulation computes the displacement field over the considered surface. The crack tip position is identified as the one that provides the best fit between the simulated and measured displacement fields. The main idea of the method is schematically shown in Figure 4. where the outer contour of T3DIC measurements, represented in green, are prescribed to the FE model, and internal nodes (blue region) are used for comparison with FE analyses. Several crack tip positions are tested along the groove region (red dashed line), and the one that gives the least root mean squared displacement gap is considered the best estimate and thus, chosen as the crack tip position for the considered image.

55 The method is then repeated for every image taken during the test. It will be referred to as FEMU henceforth. 

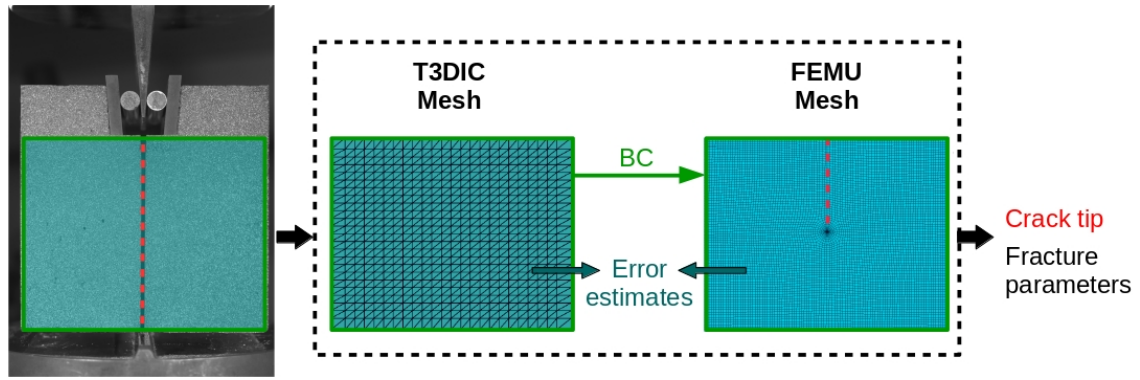

Figure 4: Adaptation of methodology introduced in Ref. [30] to the WST. An area around the propagation path is chosen and the displacements measured by T3DIC at the outer boundaries (green) are used as boundary conditions. The internal measured displacements (blue) are used for comparison with FE outputs, and the crack tip position is tested in the groove region (red).

It is important to highlight that such a procedure is run for each image taken during the experiment, so that one crack tip position is obtained for each considered step time. Within an FE code, it is generally possible to calculate quantities such as the mode I and II SIFs $K_{I}, K_{I I}$, and the $T$-stress [41. Thus the change of these fracture mechanics parameters is evaluated for each analyzed crack length. Given the thickness of the sample, a plane strain hypothesis is considered in this paper for all the reported analyses.

The FEMU displacements and mesh are exported and interpolated onto the T3DIC mesh. The nodal displacement difference between T3DIC and FE analyses is computed, and the displacement gap consists in the RMS difference

$$
\phi_{u}^{2}=\sum_{i=1}^{N}\left(v_{i}^{\mathrm{FEMU}}-v_{i}^{\mathrm{T} 3}\right)^{2},
$$

in which $v_{i}$ are nodal displacements. The superscript indicates if the displacements are obtained from FEMU or T3DIC analyses. It is worth noting that the gap estimate proposed in Equation (7) considers the same influence from every considered node. This definition may be improved using the T3DIC Hessian to augment the weight on nodes with respect to lower measurement uncertainties [42. Once different crack positions are tested, the one that provides the minimum $\phi_{u}$ is taken as the estimated crack tip position, and the fracture mechanics properties are assessed with this configuration. 
Although $\phi_{u}^{2}$ is considered for crack tip identification, the gray level residual $\rho_{\text {FEMU }}$ can be computed using the nodal displacements of the FE model $\left\{\boldsymbol{v}_{\mathrm{FE}}\right\}$

$$
\rho_{\mathrm{FEMU}}=f(\mathbf{x})-g\left(\mathbf{x}+\boldsymbol{\Psi}_{\mathrm{FE}}\left(\mathbf{x},\left\{\boldsymbol{v}_{\mathrm{FE}}\right\}\right)\right),
$$

where $\boldsymbol{\Psi}_{\mathrm{FE}}$ is the vector containing the shape functions converting nodal to pixel displacements, which is linearly dependent on the measured degrees of freedom $\left\{\boldsymbol{v}_{\mathrm{FE}}\right\}$. The corresponding global residual reads

$$
\bar{\rho}_{\mathrm{FEMU}}=\frac{R M S\left(\rho_{\mathrm{FEMU}}\right)}{\Delta f} .
$$

It will be compared with T3DIC results and the following integrated method.

\subsection{Method 2: Integrated DIC}

This section summarizes the methodology used to analyze a wedge splitting test with Integrated-DIC [27] using a closed-form solution. Williams' series [28] describe the kinematics in this case, and the gray level residual is minimized (Equation 1), instead of the displacement gap for FEMU (Equation (7)). The sought displacement field reads

$$
\mathbf{u}(\mathbf{z})=\sum_{j=I}^{I I} \sum_{n=p_{i}}^{p_{f}} \omega_{n}^{j} \boldsymbol{\psi}_{n}^{j}(\mathbf{z}),
$$

where the vector fields are defined in the complex plane

$$
\mathbf{z}=\left(x-x_{c}\right)+\left(y-y_{c}\right) i=r \exp (i \theta),
$$

where $\left(x_{c}, y_{c}\right)$ are the coordinates of the crack tip position, $j=I$ is related to the mode I (opening) regime and $j=I I$ to mode II (shearing). The amplitudes $\omega_{n}^{j}$ become the unknown kinematic degrees of freedom of IDIC. The corresponding displacement fields are described by

$\boldsymbol{\psi}_{n}^{I}=\frac{A(n)}{2 \mu \sqrt{2 \pi}} r^{n / 2}\left[\kappa \exp \left(\frac{i n \theta}{2}\right)-\frac{n}{2} \exp \left(\frac{i(4-n) \theta}{2}\right)+\left((-1)^{n}+\frac{n}{2}\right) \exp \left(-\frac{i n \theta}{2}\right)\right]$,

and

$\boldsymbol{\psi}_{n}^{I I}=\frac{i A(n)}{2 \mu \sqrt{2 \pi}} r^{n / 2}\left[\kappa \exp \left(\frac{i n \theta}{2}\right)+\frac{n}{2} \exp \left(\frac{i(4-n) \theta}{2}\right)+\left((-1)^{n}-\frac{n}{2}\right) \exp \left(-\frac{i n \theta}{2}\right)\right]$, 
where $\kappa$ is equal to $(3-\nu) /(1+\nu)$ for plane stress states or $3-4 \nu$ for plane strain states, $\nu$ the Poisson's ratio and $A(n)$ is defined by

$$
A(n)=\cos \left(\frac{n \pi}{2}\right)^{2}+\sin \left(\frac{n \pi}{2}\right)
$$

The amplitude $\omega_{1}^{j}$ gives access to Stress Intensity Factors (SIFs), the amplitude $\omega_{2}^{I}$ provides the $T$-stress (positive in compressive) and $\omega_{2}^{I I}$ the rigid body rotation. Higher order fields account for deviations from the theoretical assumption of an infinite medium [34]. With the use of two additional terms in the series, namely using $p_{i}=0, \omega_{0}^{I}$ and $\omega_{0}^{I I}$ are related to rigid body translations.

Although not usual, negative values of $p_{i}$ can help to account for nonlinearities [34, 30. It can be seen from Equations $(12$ and $(13)$ that for negative values of $n$, super-singular solutions arise near the crack tip $(r=0)$. They are also important to locate the crack tip position, especially $\omega_{-1}^{I}$. With the assumption that the crack tip is perturbed by a small distance $d$, along with some recursive properties of the Williams' series [33], it is possible to derive the offset

$$
d=\frac{2 \omega_{-1}^{I}}{\omega_{1}^{I}},
$$

which provides an estimation of crack tip shift to find the correct solution. In the sequel, $p_{i}=-3$ is taken to account for nonlinearities 34. The maximum value $p_{f}=8$ is chosen after a convergence analysis [27]. A normalization of $\boldsymbol{\Psi}$ is also performed in order to decrease floating point rounding errors.

The pacman-like ROI used for IDIC ${ }^{1}$ is shown in Figure 5 An opening of 40 pixels (pacman mouth) is taken in order not to consider the cracked mouth. The Williams' series are projected onto an FE mesh to allow the use of the same FE-DIC code as used in the previous section. This mesh is chosen sufficiently fine not to influence the results. (A convergence study was performed to check this statement.)

\footnotetext{
${ }^{1}$ This ROI is always centered about the evaluated crack tip position
} 

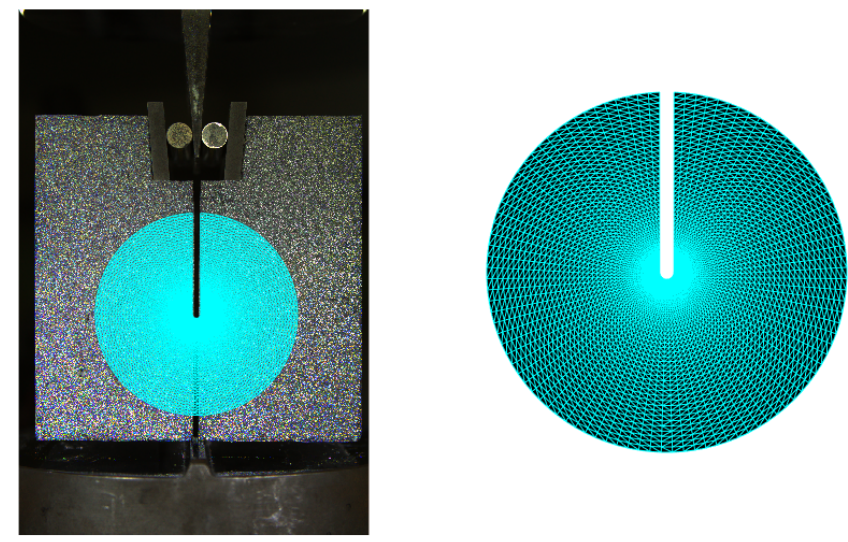

Figure 5: Example of the pacman-like mesh used in IDIC analyses.

The procedure to find the crack tip positions and the mechanical parameters of the fracture process can be summarized in the present steps:

1. A crack path is first defined as a straight line, in the groove region of the photographed sample face (Figure 4), as proved by the analysis of the maximum eigen strain fields (Figure 3);

2. The parameters defining the truncation of Williams' series are chosen, i.e., $p_{i}=-3, p_{f}=8$, the radius for normalization and mesh parameters;

3. The calculation is initialized for the crack position assumed to be located at the notch root;

4. An optimization algorithm evaluates the amplitudes $\{\boldsymbol{\omega}\}$ that decrease the global gray level residual [20;

5. The crack tip position is updated using Equation 15;

6. Steps 4 and 5 are repeated until convergence (i.e., $d<0.1$ pixel).

After convergence, the corresponding gray level residual field is stored

$$
\rho_{\mathrm{IDIC}}=f(\mathbf{x})-g\left(\mathbf{x}+\boldsymbol{\Psi}_{\mathrm{IDIC}}(\mathbf{x},\{\boldsymbol{\omega}\})\right),
$$

31 where $\{\boldsymbol{\omega}\}$ gathers all converged $\omega_{n}^{j}$ amplitudes (see Equation $10 p$ ), and $\boldsymbol{\Psi}_{\text {IDIC }}$ are the shape functions described in Equations 12 and 13 evaluated for each 
pixel position $\mathbf{x}$. From this information, the global IDIC residual becomes

$$
\bar{\rho}_{\text {IDIC }}=\frac{R M S\left(\rho_{\text {IDIC }}\right)}{\Delta f} .
$$

This methodology will be referred to as IDIC in Section 4 .

\section{Analysis of a virtual experiment}

First, a virtual experiment is considered to test both approaches on a configuration for which the exact solution is known. It is discussed how to deform a reference image with displacements obtained from numerical simulations (e.g., $\left.A b a q u s^{T M}\right)$. In the sequel, this virtually deformed image is then analyzed using the previous two methods. This virtual experiment will be referred to as VE in Section 3.2

\subsection{Virtual experiment}

A sketch following the instructions from Ref. [9] (width $=100 \mathrm{~mm}$ and height $=100 \mathrm{~mm}$ ) is performed in $A b a q u s^{T M}$, with some adjustments such as the depth of the extrusion set to $72.5 \mathrm{~mm}$ related to the sample geometry [27]. The numerical model is presented in Figure 6. Normal pressure was applied to the vertical faces onto which the rollers would apply the load. The line at the bottom of the sample does not move in the $x$ and $y$-directions. In the $z$ direction, one single point has no motion. A straight crack is added and its tip is located in the middle of the crack propagation path of the sample (i.e., only one loading step is considered). The mesh is refined around the crack tip. A $2 \mathrm{D}$ mesh is extracted from the image plane presented in Figure 6 to consider a plane strain state. $K_{I}, K_{I I}$, and the $T$-stress measurements at this plane are considered. 


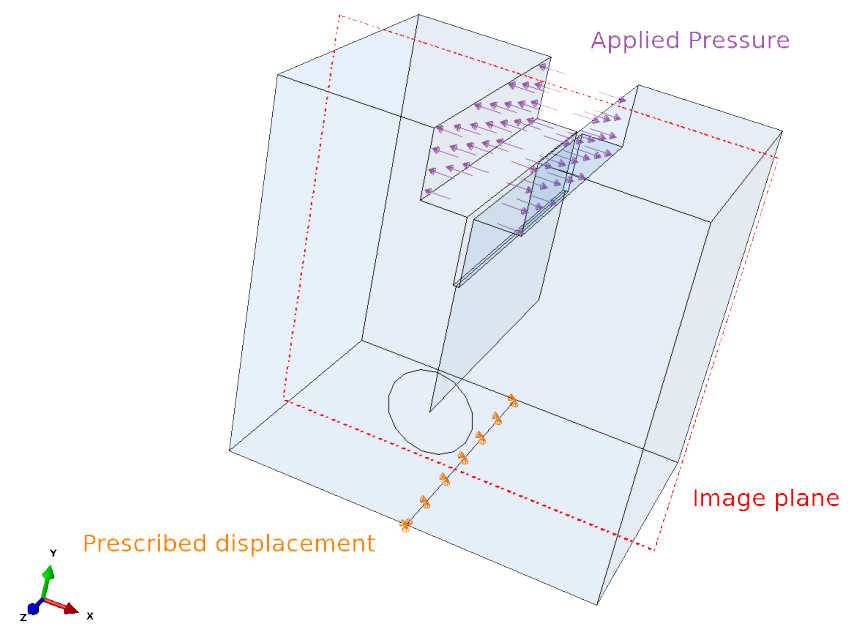

Figure 6: 3D Abaqus ${ }^{T M}$ model showing the applied pressure and the prescribed displacements. It is also possible to see the partition lines in the middle of the crack propagation path. The used image plane is also shown.

For the present study, a reference image is also required. An actual image that shows the whole sample surface 27 is used in the sequel. The 2D mesh is extracted and interpolated onto pixel coordinates such that it fits the sample in the image, as shown in Figure 7.
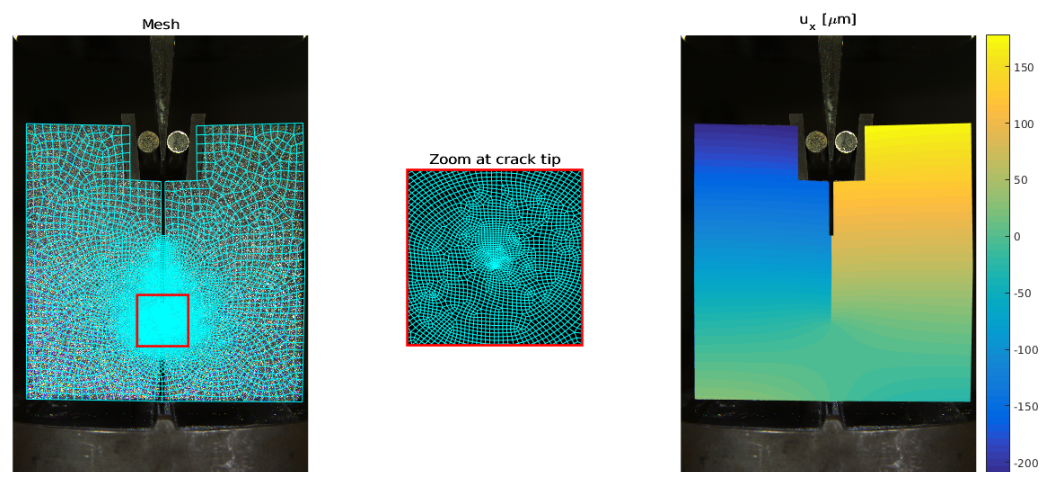

Figure 7: Superposition of 2D mesh extracted from the 3D Abaqus ${ }^{T M}$ mesh for the reference image (on the left), with a zoom about the crack tip (on the middle). Horizontal displacements $u_{x}$ (expressed in $\mu \mathrm{m}$ ) (on the right) in the virtual experiment. 
Once the displacement field for each pixel is known, the deformed image is created. In DIC the gray level conservation reads

$$
f(\mathbf{x})=g(\mathbf{x}+\mathbf{u}(\mathbf{x}))
$$

in which $\mathbf{x}$ contains integer pixel coordinates in the reference image. However the position $\mathbf{x}+\mathbf{u}(\mathbf{x})=\boldsymbol{\theta}(\mathbf{x})$ is not necessarily an integer. The evaluation of $g(\boldsymbol{\theta}(\mathbf{x}))$, which corresponds to the picture of the deformed configuration corrected by the displacement field $\mathbf{u}$, requires an interpolation scheme of the gray levels. In the present case, an inverse mapping is required, namely, integer valued positions $\mathbf{x}_{g}$ are sought to construct the picture in the deformed configuration $g$. Consequently, the position $\boldsymbol{\theta}^{-1}\left(\mathbf{x}_{g}\right)$ has to be determined for computing the gray level $f\left(\boldsymbol{\theta}^{-1}\left(\mathbf{x}_{g}\right)\right)$, which also requires an interpolation scheme [43].

To be more representative of a real experiment, it is chosen to add real noise to the deformed image. The gray level residual from T3DIC results for the first deformed image of the front face is added to the artificially deformed image. The global residual is equal to $0.57 \%$. This residual at the beginning of the test is considered to be a good approximation of the noise during the test since the load was small and no crack propagation had yet occurred.

\subsection{Results}

The results obtained from the VE analysis with different methods are gathered in Table 1 . The global residuals, $\bar{\rho}_{\text {method, }}$ in which method is replaced by VE, FEMU or IDIC, are assessed by correcting the image in the deformed configuration $g$ with the corresponding displacement fields (see Section 3.1. Equations (8) and (16), respectively). For consistency along the discussion, all the reported residuals are calculated in a pacman-shaped area centered about the crack tip position for the discussed method (see Figure 5). 
Table 1: Virtual experiment results. Calibrated fracture mechanics parameters and corresponding global gray level residuals.

\begin{tabular}{|c|c|c|c|c|c|c|c|}
\hline & $\begin{array}{c}\Delta a \\
{[\mathrm{~mm}]}\end{array}$ & $\begin{array}{c}K_{I} \\
{[\mathrm{MPa} \sqrt{\mathrm{m}}]}\end{array}$ & $\begin{array}{c}K_{I I} \\
{[\mathrm{MPa} \sqrt{\mathrm{m}}]}\end{array}$ & $\begin{array}{c}T \text {-stress } \\
{[\mathrm{MPa}]}\end{array}$ & $\begin{array}{r}\bar{\rho}_{\text {method }} \\
{[\% \Delta f]} \\
\end{array}$ & $\begin{array}{c}\bar{\rho}_{\text {method }}^{\mathrm{T} 3} \\
{[\% \Delta f]}\end{array}$ & $\begin{array}{c}\Delta \bar{\rho}_{\text {method }}^{\mathrm{T} 3} \\
{[\% \Delta f]}\end{array}$ \\
\hline VE (Sect. 3.1) & 36.0 & 3.8 & $3 \times 10^{-4}$ & 6.4 & 1.68 & 2.45 & -0.77 \\
\hline FEMU (Sect. 2.3) & 38.9 & 3.1 & $3 \times 10^{-3}$ & 7.8 & 2.52 & 2.46 & 0.06 \\
\hline IDIC (Sect. 2.4 ) & 36.4 & 3.9 & $-6 \times 10^{-2}$ & 8.8 & 2.48 & 2.45 & 0.03 \\
\hline
\end{tabular}

The fact that the residuals for the exact solution are not vanishing is due to the added noise to $g$ and gray level interpolation inaccuracies. Consequently, $\bar{\rho}_{\mathrm{VE}}$ defines the minimum level that can be achieved. Both methodologies (i.e., FEMU and IDIC) are consistent with the exact (i.e., VE) solution since the gray level residuals $\left(\bar{\rho}_{\text {FEMU }}\right.$ and $\left.\bar{\rho}_{\text {IDIC }}\right)$ are only 1.5 times higher than the minimum level $\left(\bar{\rho}_{\mathrm{VE}}\right)$, and that their difference is very small with a value that is slightly lower for IDIC. The later better predicts the crack tip position ( $\Delta a$ in Table 1) and mode I SIF for an elastic body with a single crack, while the usage of the FEMU procedures provides $T$-stress levels more accurately. Mode II SIF is believed to be close to the resolution for both methods.

Since the exact solution is known in the present case, the discussion could stop here. However, in an actual experiment, the exact solution is unknown. Since the ROIs of each method are not located at the same position in the reference image, the crack tip locations predicted by both methods are expected to be different. T3DIC will thus be used to independently assess global residuals computed over the same ROI as those in the considered methods. Since in T3DIC no mechanics-based assumptions are made on the displacement fields apart from their continuity ${ }_{2}^{2}$ the global residuals $\bar{\rho}_{\text {method }}^{\mathrm{T} 3}$ for the same ROI of each method are also evaluated. The difference in global residuals

$$
\Delta \bar{\rho}_{\text {method }}^{\mathrm{T} 3}=\bar{\rho}_{\text {method }}-\bar{\rho}_{\text {method }}^{\mathrm{T} 3}
$$

\footnotetext{
${ }^{2}$ Note that the cracked area is masked by the pacman mouth (Figure 5 )
} 
then assesses the overall identification quality $\left(i . e ., \bar{\rho}_{\text {method }}^{\mathrm{T} 3}\right.$ is thus taken as the reference) and the smaller $\bar{\rho}_{\text {method }}^{\mathrm{T} 3}$, the better the identification result.

Table 1 shows that $\bar{\rho}_{\text {method }}^{\mathrm{T} 3}$ is virtually identical for the three methods. This is expected since this virtual case only involves noise and gray level interpolation inaccuracies. The fact that $\bar{\rho}_{\mathrm{VE}}^{\mathrm{T} 3}$ is greater than $\bar{\rho}_{\mathrm{VE}}$ is due to the T3DIC mesh that is rather coarse. This choice was made since very small displacements are sought (see below) and a finer mesh would have induced higher measurement uncertainties 44 . This choice also explains why $\Delta \bar{\rho}_{\mathrm{VE}}^{\mathrm{T} 3}<0$. Had a finer mesh been used, T3DIC would be expected to be closer to the VE solution. Furthermore, $\bar{\rho}_{\mathrm{FEMU}}^{\mathrm{T} 3}$ is slightly higher than $\bar{\rho}_{\text {IDIC }}^{\mathrm{T} 3}$, and more importantly, IDIC is closer to T3DIC than FEMU (i.e., $\Delta \bar{\rho}_{\mathrm{IDIC}}^{\mathrm{T} 3}=0.03 \%$ in comparison with $\left.\Delta \bar{\rho}_{\mathrm{FEMU}}^{\mathrm{T} 3}=0.06 \%\right)$. The difference between both methods remains very small, which validates both procedures. However, IDIC slightly outperformed FEMU in the present analysis.

\section{Experimental study}

The two methodologies described in Sections 2.3 and 2.4 are now applied to one wedge splitting test. Figure 8 shows the crack tip position for the first part of the experiment during which the crack has propagated. $\Delta a=0$ considers the crack tip to be located at the pre-notch root. Both methodologies have high uncertainties for the first two cycles, which are related to very small displacement ranges (i.e., 0.15 pixel, or $9 \mu \mathrm{m}$ at the most). It is observed that FEMU identifies smaller crack lengths than IDIC. It is worth noting that both methodologies predict different crack propagation histories on the two analyzed faces and that, in the end, the crack propagated farther on the back face (Figure 3). 

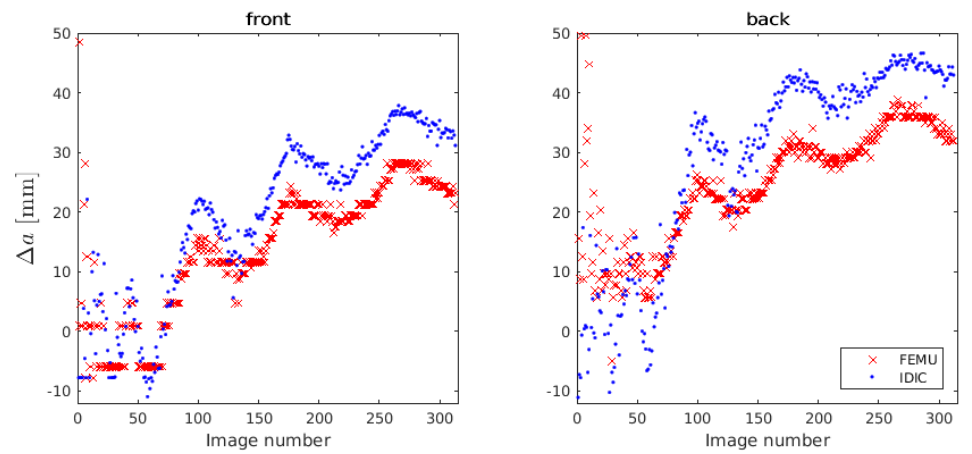

Figure 8: Crack tip position for front and back faces with both methodologies for the first 312 images acquired during the analyzed wedge splitting test.

Two features in Figure 8 need to be further commented. First, the negative values of $\Delta a$ at the beginning of the experiment, namely, a crack tip position out of the sample, and second, the crack closure in the unloading phase of each cycle. These observations do not mean that crack healing occurs in the present case. It is believed that the negative values are due to the uncertainties associated with crack tip positioning when the displacement levels are very small (i.e., at the beginning of the experiment). The crack tip itself is a feature associated with the considered fracture mechanics model (here defined with Williams' series or finite element simulations). Although the main conclusions of this work will not be affected, physically, crack propagation is believed to be stopped during unloading phases, and restarts once it reached a critical SIF level in the subsequent loading cycle.

The SIFs are reported in Figure 9. For this case in which a single macrocrack propagates guided by the groove (Figure 3), pure mode I is expected and is confirmed by both approaches. As $K_{I I}$ is close to zero, it can be used as an evaluation of the resolution for SIF evaluations. The RMS of $K_{I I}$ values measured by both methodologies and at both faces is of the order of $3 \times 10^{-2} \mathrm{MPa} \sqrt{\mathrm{m}}$. The general tendency observed for $K_{I}$ is opposite in comparison with estimates of crack tip positions, namely, lower $K_{I}$ levels and larger crack lengths are reported by IDIC in comparison to FEMU results. However, crack tip positions 
and SIFs values obtained by both techniques are of the same order of magnitude.
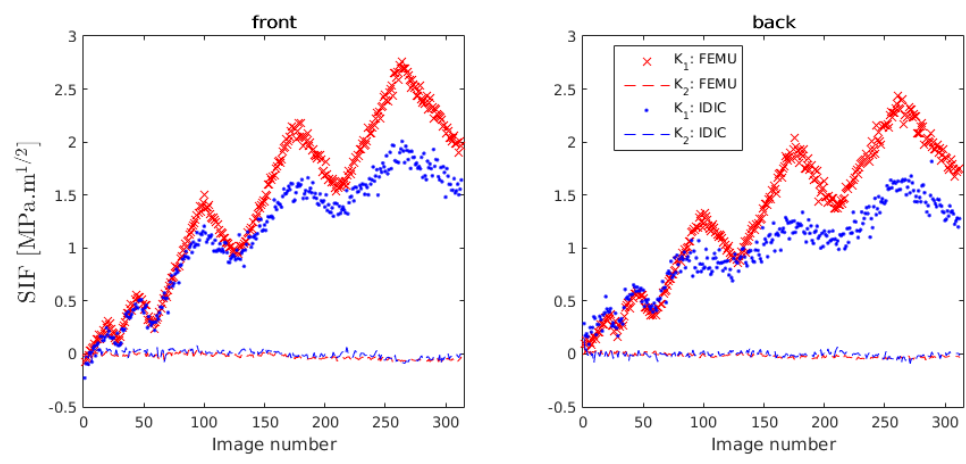

Figure 9: Mode I and II SIF histories for both faces with both approaches.

The $T$-stress histories are shown in Figure 10. The levels obtained via IDIC are generally higher, predominantly after the second cycle and for the front face. For the back face, the values are closer. The fluctuations could be related to mechanical features since some fluctuation were also observed on the load vs. crack mouth opening displacement curve for this test [27, but further studies are needed to confirm this hypothesis.
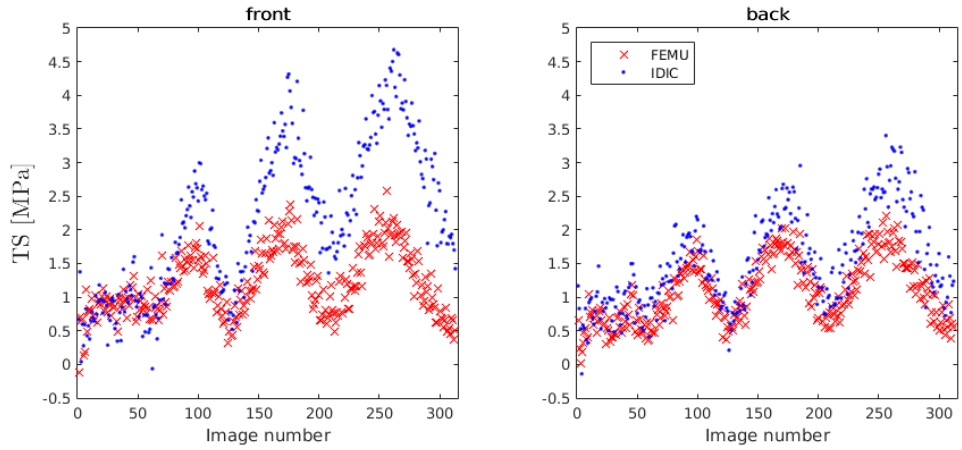

Figure 10: $T$-stresses measured with both methodologies for both faces.

In order to have an absolute evaluation of the quality of both approaches $\bar{\rho}_{\text {FEMU }}$ and $\bar{\rho}_{\text {IDIC }}$ are reported. This type of analysis is no longer an inter comparison, but probes the individual merit of each technique with respect to the pictures acquired during the experiment with the same number of pixels. The 
RMS gray level residuals are reported in Figure 11. The overall levels remain very small for the whole sequence, which validates the reported results. For both cases, increasing residuals occur with crack propagation. The longer the crack, the bigger the fracture process zone and the assumption of an elastic medium is presumably less true. Further, 3D effects may also be more pronounced [45], which make the 2D assumptions used in Williams' series and FE calculations less accurate.
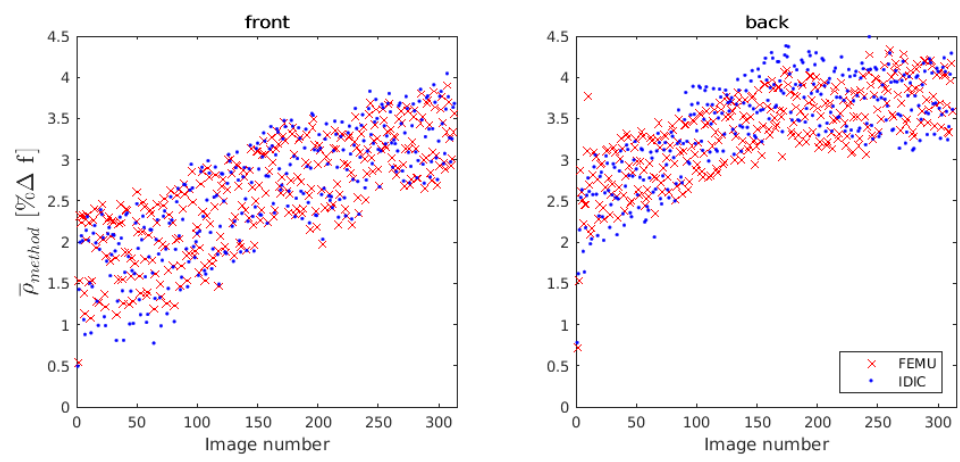

Figure 11: Dimensionless residuals for the two methods (FEMU and IDIC) and both faces.

To compare in a more quantitative way the two procedures the residual difference, $\Delta \bar{\rho}$, is computed

$$
\Delta \bar{\rho}=\bar{\rho}_{\mathrm{IDIC}}-\bar{\rho}_{\mathrm{FEMU}}
$$

Plots of $\Delta \bar{\rho}$ for both faces of the sample are shown in Figure 12 The differences are in $0.2 \%$ range, which is lower than the acquisition noise for these images (i.e., $\approx 0.6 \%$ ). However, IDIC shows lower residuals at the beginning the test. The crack tip position is better captured (Figure 8), even though the SIF levels are rather consistent with both approaches (Figure 9). The fact that IDIC becomes less accurate than FEMU at the end of the propagation step may be related to the Williams' series not describing the boundary effects as the crack tip approaches the sample edge. 

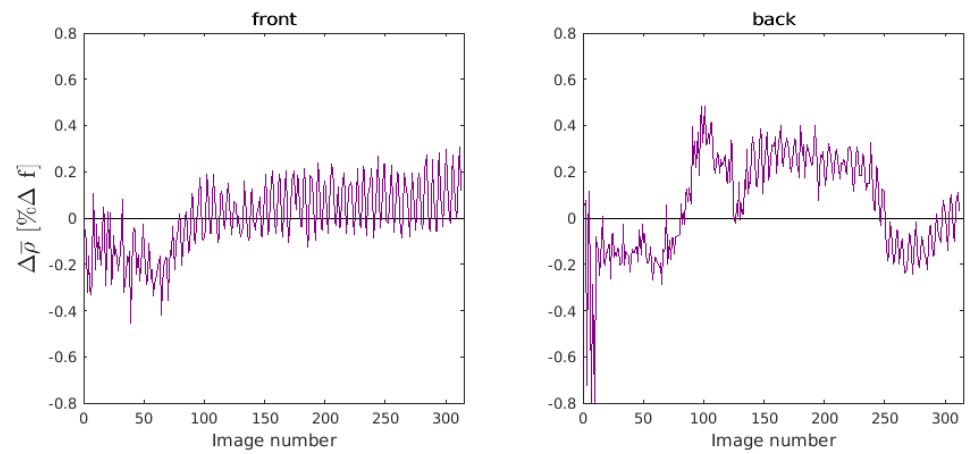

Figure 12: Residual difference for both faces. A negative (resp. positive) value corresponds to a more faithful evaluation via IDIC (resp. FEMU).

The residuals reported in Figure 11 and 12 were calculated for the same ROI size but centered about different crack tip positions (Figure 8). In a virtual experiment $\bar{\rho}_{\text {method }}^{\mathrm{T} 3}$ does not change considerably in different regions as reported in Table 1. In an actual experiment, not only slightly different image features at each ROI but also images artifacts such as lighting changes and lens focus may affect $\bar{\rho}_{\text {method }}^{\text {T3 }}$. The residual difference $\Delta \bar{\rho}_{\text {method }}^{T 3}$ (see Equation $(19)$ ) is shown in Figure 13 and indicates how close each method was close to T3DIC. IDIC residuals are closer to T3DIC than FEMU. Small negative values seen for IDIC and FEMU in some images indicate that they outperformed T3DIC, which can be explained by the non-optimal T3DIC mesh. Although the residuals for both methodologies are in the same range (Figure 12), the ROIs in which FEMU converged were presumably less affected by image artifacts and the residuals were farther from T3DIC residuals. 

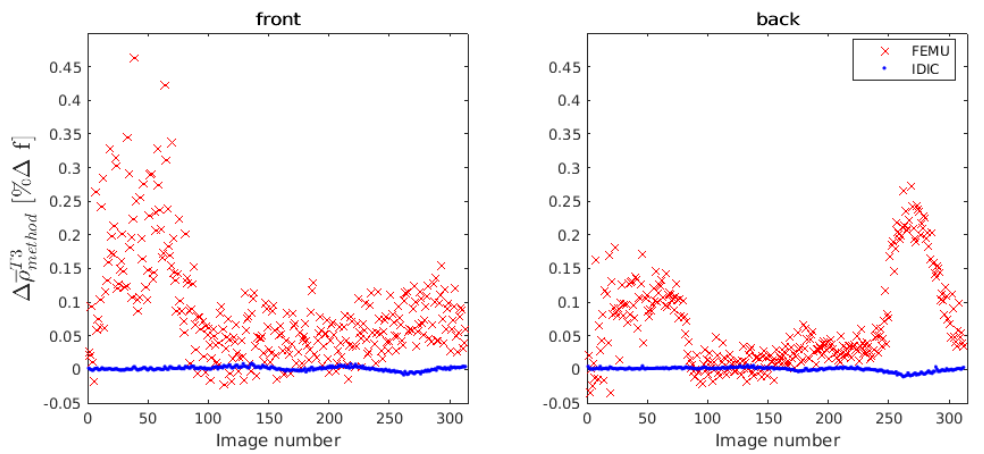

Figure 13: Differences from IDIC and FEMU residuals in comparison with T3DIC. Positive values are related to smaller residuals measured with T3DIC.

The main conclusion when considering different regions separately (Figure11 and 12 is that IDIC was performing better at the very beginning and then FEMU would be preferred for the rest of the test. However, using $\bar{\rho}_{\text {method }}^{\text {T3 }}$ as a reference (Figure 13) in order to account for image artifacts and textures on different regions, the conclusion is that, for the present case, IDIC is more accurate for the whole analysis.

Last, even though very small, $K_{I I}$ is considered to analyze the R-curve behavior defined as

$$
R=\frac{K_{I}^{2}+K_{I I}^{2}}{E}\left(1-\nu^{2}\right)
$$

in a plane strain state, as considered in FEMU and IDIC. It is worth noting that although some deviation from linear elasticity may occur, the effective crack tip is measured with full-field approaches accounting for elasticity. In such cases, with the effective instead of visible crack tips, Equation (21) can be applied [46].

The R-curve is then plotted as a function of crack tip position in Figure 14. It shows that energy consumption increases as the crack propagates (with a steeper slope for FEMU), which is related to extrinsic toughening mechanisms such as crack branching, microcracking or bridges between aggregates [47. In the present case, it was checked that crack branching was not occurring out of the groove in the photographed surface of sample, at the scale of DIC measurements (see Figure 3), namely, of the order of the element length (i.e., $\approx 0.5 \mathrm{~mm}$ ). 
With the chosen magnification, the main purpose of DIC was to analyze the macroscopic fracture mechanics parameters, i.e., R-curve behavior, and could resolve the effect of the toughening mechanisms occurring at lower scales. The observed R-curve behavior may indicate crack branching, and although not seen on the strain fields reported in Figure 3, it may take place in the bulk of the material. For checking such mechanisms, in situ tomographies analyzed with Digital Volume Correlation would be a suitable approach [48.
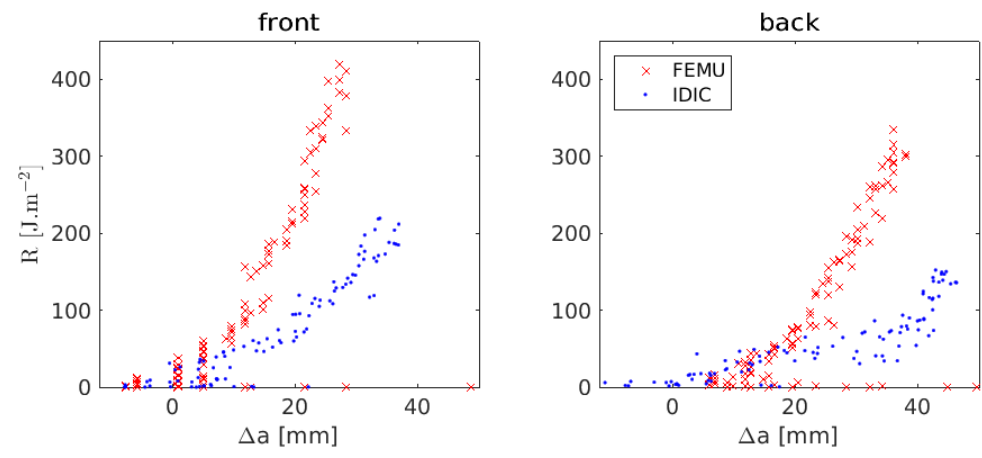

Figure 14: $\mathrm{R}$ vs. crack length for both faces with both approaches. Images taken during unloading steps are not accounted for the computation of $R$.

The present study enables to assess uncertainties associated with the use of two different identification techniques. The latter ones provided results that were both considered as realistic and may be used when comparing crack propagation in different materials compositions, for instance. However, they led to significant differences in the crack propagation resistance curve of the investigated material (Figure 14). Thanks to the use of gray level residuals, the merit of both techniques could be assessed, but more importantly it could be decided which one was more trustworthy (i.e., IDIC in the present case).

In order to validate the R-curves reported in Figure 14, a last study is performed. First, the loading history shown in Figure 2 may be integrated to estimate the fracture energy, $\gamma_{w o f}(v)$

$$
\gamma_{w o f(v)}^{\operatorname{method}}=\frac{1}{2 A^{\text {method }}} \int_{\Delta h_{0}}^{\Delta h_{f}} F_{v} d(\Delta h)
$$


where the interval $\left[\Delta h_{0}, \Delta h_{f}\right]$ corresponds to the loading envelope and the final unloading, $\Delta h$ is the vertical displacement of the actuator, $F_{v}$ the vertical force, and method is substituted by IDIC or FEMU for the evaluation of the cracked area $A$. The latter is considered as the maximum crack length $\left(\Delta a_{\max }\right.$ for last point before final unloading) obtained for the method (IDIC or FEMU) multiplied by the thickness $t_{g}$ of the specimen inside the groove (i.e., $65 \mathrm{~mm}$ ). It is worth noting that $\gamma_{w o f(v)}$ corresponds to an upper bound since it includes dissipated energy through friction of the loading parts.

The splitting force $F_{h}$ vs. displacement $\Delta \delta$ curves are shown in Figure 15 in which the splitting displacement $\Delta \delta$ is measured from T3DIC results at the locations shown in Figure 1 namely, at the same height as the splitting forces are applied.

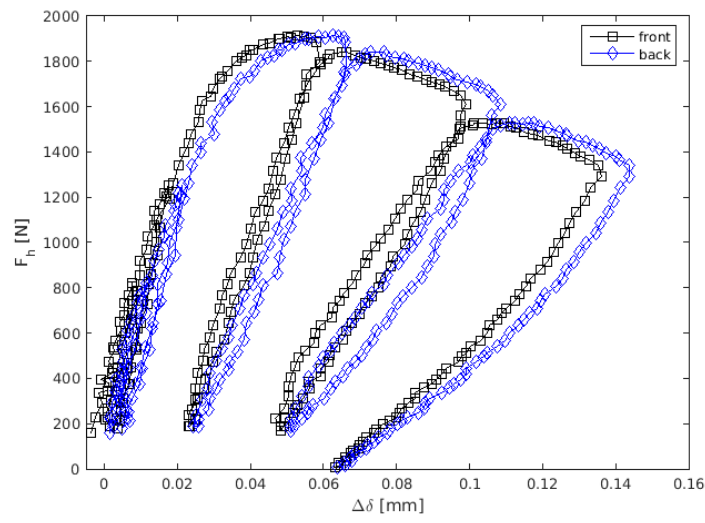

Figure 15: Splitting force (i.e., 5.715 times the vertical force) versus splitting displacement $\Delta \delta$ measured at the locations shown in Figure 1

«36 By integrating the data shown in Figure 15, the fracture energy $\gamma_{w o f}$ reads

$$
\gamma_{w o f}^{\text {method }}=\frac{1}{2 A^{\text {method }}} \int_{\Delta \delta_{0}}^{\Delta \delta_{f}} F_{h} d(\Delta \delta)
$$

where the interval $\left[\Delta \delta_{0}, \Delta \delta_{f}\right]$ corresponds to the loading envelope and the final unloading. In the present case, the DIC extensometry only accounts for the work performed on the specimen itself, thus is more representative of the work 
of fracture of the studied material.

To calculate the average of $R$, which is denoted by $\bar{R}$, for IDIC and FEMU the R-curves shown in Figure 14 are integrated

$$
\bar{R}^{\text {method }}=\frac{1}{\Delta a_{\max }} \int_{\Delta a_{0}}^{\Delta a_{\max }} R^{\text {method }} d\left(\Delta a^{\text {method }}\right)
$$

Since the dissipated energy is consumed to create two cracked surfaces, $\bar{R}=$ $2 \gamma_{w o f}(h)$ 49.

The calculated energies are listed in Table 2, All reported values are expected to underestimate the fracture energy measured for the full crack propagation (with same methodology), which is not accessible in the experiment reported herein. The fracture energies have the same order of magnitude, which is a further validation of the R-curves predicted with IDIC and FEMU. However, IDIC predicts values that are closer to those obtained with DIC extensometry than FEMU. FEMU even provides estimates above the upper bound (i.e., $\left.\gamma_{w o f(v)}\right)$.

Table 2: Fracture energies (expressed in $\mathrm{J} / \mathrm{m}^{2}$ ) calculated with different approaches.

\begin{tabular}{|c|c|c|c|c|}
\hline & Fract. energy & front & back & mean \\
\hline \multirow{3}{*}{ FEMU } & $2 \gamma_{\text {wof }(v)}$ & 114 & 83 & 99 \\
& $2 \gamma_{w o f}$ & 92 & 71 & 82 \\
& $\bar{R}$ & 162 & 97 & 130 \\
\hline \multirow{2}{*}{ IDIC } & $2 \gamma_{w o f}(v)$ & 85 & 69 & 77 \\
& $2 \gamma_{w o f}$ & 69 & 60 & 64 \\
& $\bar{R}$ & 84 & 52 & 68 \\
\hline
\end{tabular}

Even though FEMU and IDIC results are in the same range for the various quantities reported in the present section, IDIC results are more consistent with experimental data in terms of gray level residuals, but even more importantly when compared with independent estimates of fracture energies. For the latter ones, it is shown that about $20 \%$ is dissipated by friction when the upper bound estimate is compared with that derived from the splitting force vs. displacement data, or equivalently by R-curves provided by IDIC. 


\section{Conclusions}

The FEMU methodology 30] was adapted to analyze WSTs. An automated procedure was implemented to create an FE model with Dirichlet boundary conditions measured via T3DIC analyses. The crack tip position was identified, among several tested positions, as the one that provided the best kinematic fit. Interaction integrals of the FE code were used to estimate fracture-related properties (i.e., SIFs and T-stress). This approach was compared with IDIC when applied to a WST [27]. First, a virtual experiment was analyzed, then followed by experimental analyses for both faces of a refractory castable specimen.

FEMU allows for better $T$-stress estimates, while IDIC is more trustworthy for estimating the crack tip position and mode I SIF in the virtual experiment. Gray level residuals were used to check the merit of each technique directly with experimental pictures. FEMU residuals were farther from T3DIC than IDIC when considering T3DIC as the reference to check the quality of measurements, proving that IDIC is slightly more trustworthy than FEMU in the present case.

IDIC provides longer crack lengths coupled with lower SIFs in comparison with FEMU. Consequently, the R-curve behavior is less steep for the former. Different levels of properties on each side and measured by each method highlights the importance of such analyses performed on both sample faces. These differences are related to experimental imperfections such as the fine alignment of the wedge and microstructural heterogeneities.

Accessing gray level residuals is a powerful tool to check analyses with the experimental data when the actual solution is not known. It is of utmost importance to consider the measurement regions in such analyzes. IDIC residuals were closer to T3DIC resulting in more reliable measurements.

The evaluations of fracture energies were consistent with both discussed methods, namely, the same order of magnitude of the R-curves was obtained via IDIC and FEMU. When compared with FEMU, IDIC estimates were closer to those based on DIC extensometry. While IDIC was comparable to conventional methods, FEMU resulted in fracture energies greater than the experimental 
upper bound. All these differences show that the estimation of the crack tip location, which was different for both investigated approaches, has to be very accurate. The use of the first supersingular field in Williams' series for estimating the crack tip location via IDIC is further validated thanks to the present study.

\section{Acknowledgments}

JAR thanks CNPq for the productivity scholarship, grant \#307127/2013-3.

RV's stay at LMT was supported through an RIA scholarship, grant \#2017/209119, São Paulo Research Foundation (FAPESP).

\section{References}

[1] Lee WE, Vieira W, Zhang S, Ghanbari Ahari K, Sarpoolaky H, Parr C. Castable refractory concretes. International Materials Reviews 2001;46(3):145-67.

[2] Luz AP, Braulio MAL, Pandolfelli VC. Refractory Castable Engineering; vol. 1. 1 ed.; São Carlos, SP: Göller Verlag; 2015.

[3] Lee WE, Moore RE. Evolution of in situ refractories in the 20th century. Journal of the American Ceramic Society 1998;81(6):1385-410.

[4] Studart AR. Bioinspired ceramics: Turning brittleness into toughness. Nature materials 2014;13(5):433-5.

[5] Wachtman J. Materials and Equipment - Whitewares - Refractory Ceramics - Basic Science: Ceramic Engineering and Science Proceedings, Volume 16. No. 1 in Ceramic Engineering and Science Proceedings; Wiley; 2009. ISBN 9780470316306.

[6] Tschegg E. Prüfeinrichtung zur Ermittlung von bruchmechanishen Kennwerten sowie hiefür geeignete, Prüfkörper, Austrian Pat. AT 390328B, registered. 1986. 
[7] Brühwiler E, Wittmann FH. The wedge splitting test, a new method of performing stable fracture mechanics tests. Engineering Fracture Mechanics 1990;35(1-3):117-25.

[8] Harmuth H. Stability of crack propagation associated with fracture energy determined by wedge splitting specimen. Theoretical and Applied Fracture Mechanics 1995;23:103-8.

[9] Ribeiro S, Rodrigues JA. The influence of microstructure on the maximum load and fracture energy of refractory castables. Ceramics International 2010;36(1):263-74.

[10] Grasset-Bourdel R, Alzina A, Huger M, Chotard T, Emler R, Gruber D, et al. Tensile behaviour of magnesia-spinel refractories: Comparison of tensile and wedge splitting tests. Journal of the European Ceramic Society 2013;33(5):913-23.

[11] Liao N, Li Y, Wang Q, Zhu T, Jin S, Sang S, et al. Synergic effects of nano carbon sources on thermal shock resistance of $\mathrm{Al}_{2} \mathrm{O}_{3}-\mathrm{C}$ refractories. Ceramics International 2017;43(16):14380-8.

[12] Long B, Xu G, Buhr A, Jin S, Harmuth H. Fracture behaviour and microstructure of refractory materials for steel ladle purging plugs in the system $\mathrm{Al}_{2} \mathrm{O}_{3}-\mathrm{MgO}-\mathrm{CaO}$. Ceramics International 2017;43(13):9679-85.

[13] Zhu T, Li Y, Sang S, Xie Z. Fracture behavior of low carbon $\mathrm{MgO}-\mathrm{C}$ refractories using the wedge splitting test. Journal of the European Ceramic Society 2017;37(4):1789-97.

[14] Zhu T, Li Y, Sang S, Xie Z. Mechanical behavior and thermal shock resistance of $\mathrm{MgO}-\mathrm{C}$ refractories: Influence of graphite content. Ceramics International 2017;43(9):7177-83.

[15] Jin S, Gruber D, Harmuth H. Determination of Youngs modulus, fracture energy and tensile strength of refractories by inverse estimation of a wedge splitting procedure. Engineering Fracture Mechanics 2014;116:228 -36. 
[16] Sutton MA. Computer vision-based, noncontacting deformation measurements in mechanics: A generational transformation. Applied Mechanics Reviews 2013;65(AMR-13-1009, 050802).

[17] McNeill S, Peters W, Sutton M. Estimation of stress intensity factor by digital image correlation. Engineering Fracture Mechanics 1987;28(1):10112.

[18] Abanto-Bueno J, Lambros J. Investigation of crack growth in functionally graded materials using digital image correlation. Engineering Fracture Mechanics 2002;69:1695-711.

[19] Forquin P, Rota L, Charles Y, Hild F. A method to determine the toughness scatter of brittle materials. International Journal of Fracture 2004;125(1):171-87.

[20] Roux S, Hild F. Stress intensity factor measurements from digital image correlation: post-processing and integrated approaches. International Journal of Fracture 2006;140(1-4):141-57.

[21] Mathieu F, Hild F, Roux S. Identification of a crack propagation law by digital image correlation. International Journal of Fatigue 2012;36(1):14654 .

[22] Saracura RGM, Canto RB, Pandolfelli VC, Schmitt N, Hild F. Surface crack network detection on $\mathrm{MgO}$-based refractory castable by digital image correlation. China's Refractories 2015;24(1):32-7.

[23] Belrhiti Y, Pop O, Germaneau A, Doumalin P, Dupré JC, Harmuth H, et al. Investigation of the impact of micro-cracks on fracture behavior of magnesia products using wedge splitting test and digital image correlation. Journal of the European Ceramic Society 2015;35(2):823-9.

[24] Dai Y, Gruber D, Harmuth H. Observation and quantification of the fracture process zone for two magnesia refractories with different brittleness. Journal of the European Ceramic Society 2017;37(6):2521-9. 
[25] Dai Y, Gruber D, Harmuth H. Determination of the fracture behaviour of $\mathrm{MgO}$-refractories using multi-cycle wedge splitting test and digital image correlation. Journal of the European Ceramic Society 2017;37(15):5035-43.

[26] Dupré JC, Doumalin P, Belrhiti Y, Khlifi I, Pop O, Huger M. Detection of cracks in refractory materials by an enhanced digital image correlation technique. Journal of Materials Science 2018;53(2):977-93.

[27] Vargas R, Neggers J, Canto RB, Rodrigues JA, Hild F. Analysis of wedge splitting test on refractory castable via integrated DIC. Journal of the European Ceramic Society 2016;36(16):4309-17.

[28] Williams ML. On the stress distribution at the base of a stationary crack. Journal of Applied Mechanics 1957;24(1):109-14.

[29] Rieder KA, Tschegg EK, Harmuth H. Notch sensitivity of ordinary ceramic refractory materials. Journal of Materials Science Letters 1998;17(8):675-8.

[30] Mathieu F, Aimedieu P, Guimard JM, Hild F. Identification of interlaminar fracture properties of a composite laminate using local full-field kinematic measurements and finite element simulations. Composites Part A: Applied Science and Manufacturing 2013;49:203-13.

[31] Affagard JS, Mathieu F, Guimard JM, Hild F. Identification method for the mixed mode interlaminar behavior of a thermoset composite using displacement field measurements and load data. Compos Part A 2016;91:238-49.

[32] Grédiac M, Hild F. Full-field measurements and identification in solid mechanics. John Wiley \& Sons; 2012.

[33] Roux S, Réthoré J, Hild F. Digital image correlation and fracture: An advanced technique for estimating stress intensity factors of $2 \mathrm{D}$ and $3 \mathrm{D}$ cracks. Journal of Physics D: Applied Physics 2009;42:214004.

[34] Henninger C, Roux S, Hild F. Enriched kinematic fields of cracked structures. International Journal of Solids and Structures 2010;47(24):3305-16. 
[35] Diógenes HJF, Cossolino LC, Pereira AHA, El Debs MK, El Debs ALHC. Determination of modulus of elasticity of concrete from the acoustic response. Revista IBRACON de Estruturas e Materiais 2011;4(5):803-13.

[36] Pereira AHA, Miyaji DY, Cabrelon MD, Medeiros J, Rodrigues JA. A study about the contibution of the $\alpha-\beta$ phase transition of quartz to thermal cycle damage of a refractory used in fluidized catalytic cracking units. Cerâmica 2014;60:449-56.

[37] Miyaji DY, Otofuji CZ, Cabrelon MD, Medeiros J, Rodrigues JA. The coke effect on the fracture energy of a refractory castable for the petrochemical industry. In: Proceedings of the Unified International Technical Conference on Refractories (UNITECR 2013). Wiley Online Library; 2014, p. 1111-6.

[38] Hild F, Roux S. Digital image correlation. In: Rastogi P, Hack E, editors. Optical Methods for Solid Mechanics. A Full-Field Approach. Weinheim (Germany): Wiley-VCH; 2012, p. 183-228.

[39] Besnard G, Hild F, Roux S. "Finite-Element" displacement fields analysis from digital images: Application to Portevin-Le Chatelier bands. Experimental Mechanics 2006;46(6):789-803.

[40] Leclerc H, Périé J, Roux S, Hild F. Integrated digital image correlation for the identification of mechanical properties; vol. LNCS 5496. Berlin (Germany): Springer; 2009, p. 161-71.

[41] Simulia . Contour integral evaluation (section 11.4.2). 2010,.

[42] Mathieu F, Leclerc H, Hild F, Roux S. Estimation of elastoplastic parameters via weighted FEMU and integrated-DIC. Experimental Mechanics 2015;55(1):105-19.

[43] Réthoré J, Muhibullah, Elguedj T, Coret M, Chaudet P, Combescure A. Robust identification of elasto-plastic constitutive law parameters from digital images using 3D kinematics. International Journal of Solids and Structures 2013;50(1):73-85. 
[44] Hild F, Roux S. Digital Image Correlation. Weinheim (Germany): WileyVCH; 2012, p. 183-228.

[45] Réthoré J, Roux S, Hild F. Optimal and noise-robust extraction of fracture mechanics parameters from kinematic measurements. Eng Fract Mech 2011;78(9):1827-45.

[46] Bornhauser A, Kromp K, Pabst RF. R-curve evaluation with ceramic materials at elevated temperatures by an energy approach using direct observation and compliance calculation of the crack length. Journal of Materials Science 1985;20(7):2586-96.

[47] Launey ME, Ritchie RO. On the fracture toughness of advanced materials. Advanced Materials 2009;21(20):2103-10.

[48] Hild F, Bouterf A, Roux S. Damage measurements via DIC. International Journal of Fracture 2015;191(1-2):77-105.

[49] Sakai M, Bradt RC. Fracture toughness testing of brittle materials. International Materials Reviews 1993;38(2):53-78. 